\title{
D Preoperative Considerations in Patients with Advanced CrossMark Liver Disease
}

\author{
Reza Aminnejad ${ }^{1}$, Faezeh Alemi², Saeid Safari³ ${ }^{3}$ Ahmad Hormati², 4, ${ }^{*}$, Mohammad Reza Ghadir ${ }^{2}$ \\ Mohammad Saeedi ${ }^{1}$, Mahboobeh Afifian ${ }^{5}$
}

1. Department of Anesthesiology, Qom University of Medical Sciences, Qom, Iran.

2. Gastroenterology and Hepatology Disease Research Center, Qom University of Medical Sciences, Qom, Iran.

3. Pain Research Center, Iran University of Medical Sciences, Tehran, Iran.

4. Gastrointestinal and Liver Disease Research Center, Firoozgar Hospital, Iran University of Medical Sciences, Tehran, Iran

5. MSc of Health Information Technology of Tehran University of Medical Sciences, Tehran, Iran

\section{* Corresponding Author:}

Ahmad Hormati, MD

Gastroenterology and Hepatology Disease Research Center, Shahid Beheshti Hospital, Qom, Iran.

Telefax: + 982536122053

Email: hormatia@yahoo.com

Received: 02 Jun. 2019

Accepted: 05 Sep. 2019
Please cite this paper as:

Aminnejad R, Alemi F, Safari S, Hormati A, Saeedi M, Afifian M. Preoperative Considerations in Patients with Advanced Liver Disease. Middle East J Dig Dis 2019; 11:237-239. doi:10.15171/mejdd.2019.156.

\section{BACKGROUND}

Liver cirrhosis is defined as the advanced stage of liver disease, which presents with loss of normal hepatic tissue and its replacement by fibrotic tissue. It leads to alteration in all liver functions including metabolic, synthetic, and coagulative function. Besides, accumulation of toxins may alter mental status. This pathogenesis alters the metabolism of many drugs, so that they should be used carefully or will need dose adjustment. ${ }^{1}$

A necessity for anesthesia and surgery, for liver related or unrelated indications, is a challenging issue in the management of patients with cirrhosis. This would be the art of a gastroenterologist to assess the present situation of the patient and weigh the risks and benefits to help the anesthesiologist and the surgeon in making the best plan. ${ }^{2}$

The final decision will be made upon the liver disease situation and the necessity and urgency of the surgical procedure. However, even in an acceptable anesthesiological and surgical risk background, considering some points before, during, and after the procedure is necessary to minimize the complications.

\section{Pre-operative visit and general risk assessment}

Child-Paugh classification and model for end-stage liver disease (MELD) score, are good tools to predict the patients' peri-operative morbidity and mortality. In this era, patients who are classified as child $\mathrm{A}$, are at a moderate risk and may undergo elective surgical procedures with special intra-operative care. On the other hand, patients classified as Child $\mathrm{C}$ or with a MELD score greater than 20, are at a high risk for anesthesia, and elective surgeries are contraindicated in this group. ${ }^{3}$

Anesthesia and surgical interventions should be avoided in patients with acute viral hepatitis, fulminant hepatitis, or patients with coagulopathy. Some extra-hepatic complications including acute renal failure, cardiomyopathy, and hypoxemia should be kept in mind as contraindications of elective surgeries too. Male sex and presence of ascites are independent factors that accompany with a greater risk of morbidity and mortality. ${ }^{4}$ 


\section{Specific Considerations}

The underlying liver disease, pose the patients at some special disturbance, which should be evaluated in the preoperative consultation and corrected as much as possible. These disturbances can be listed as coagulopathy, previous history of variceal bleeding, neuropsychiatric disorders, cardiomyopathy, hepatopulmonary syndrome, and electrolyte disturbance. ${ }^{5}$

One of the most prevalent surgically important abnormalities in patients with cirrhosis is coagulopathy. Coagulation management in this specific population is not quite different from other groups and in addition to the proper use of blood and blood products, pharmacological treatment of hepatic-associated coagulopathy can be achieved by means of drugs such as aminocaproic acid, tranexamic acid, conjugated estrogen, and activated recombinant factor VII. Thromboelastography may be helpful in identifying the cause of coagulopathy and can guide the administration of coagulation products. ${ }^{4}$

In patients with positive history of bleeding from gastroesophageal varices, special attention to the global state of the patient and essential need for surgery is appreciated. Any treatable condition prior to elective surgery would be indicated. Beta blockers such as propranolol or nadolol in these patients should be continued until the surgery day. ${ }^{5}$

Neuropsychiatric changes in these patients could be the result of hepatic encephalopathy, which needs meticulous attention and long term management. In this situation need for surgery should be re-evaluated. ${ }^{6}$

In the presence of hyper-dynamic circulation, cardiologic consultation will be logical. Cirrhosis may also be associated with cardiomyopathy or diastolic dysfunction, so intraoperative management of hemodynamics can be challenging. ${ }^{7}$

Presence of dyspnea and hypoxemia, which may worsen in the upright position, could be clues for hepatopulmonary syndrome. Echocardiography in this situation could differentiate between intrapulmonary and intra-cardiac shunting. Other causes of respiratory disturbances in patients with cirrhosis include pulmonary hypertension and pleural effusion. Besides, presence of underlying pulmonary diseases including chronic obstructive pulmonary disease (COPD) should be kept in mind as a differential diagnosis. Severe hypoxemia
$\left(\mathrm{PO}_{2}<60 \mathrm{mmHg}\right)$ is a relative contraindication for surgery, excluding liver transplantation, in patients with hepatopulmonary syndrome; as the only definitive treatment for severe hepatopulmonary syndrome is liver transplantation. Administration of somatostatin and supplemental oxygen is used as supportive therapy until a donor organ becomes available. ${ }^{8}$

Electrolyte disturbances could be seen in patients with liver failure. So, preoperative evaluation and correction of any correctable condition is recommended. In extreme conditions hepatorenal syndrome could be seen, which mandates renal replacement therapy. Short term mortality rate is high in these patients and need for elective surgeries should be reassessed. ${ }^{5}$

\section{Key points about the altered metabolism of drugs in patients with cirrhosis}

Presence of liver failure mandates lowering drug dosages to minimal effective level. Whenever possible it is prudent to manage anesthesia by regional or neuraxial methods such as spinal or epidural anesthesia. Presence of coagulopathy should be kept in mind prior to some types of regional anesthesia specially neuraxial ones . ${ }^{4}$

Regarding the changes in pharmacokinetics of drugs in patients with liver failure, considering the half-life prolongation of some premedications and induction drugs has clinical importance. The half-life of some routinely used drugs, including benzodiazepines and lidocaine increases significantly, which needs dose adjustment. Due to hypo-albuminemia, the volume of distribution of the drugs that routinely bind to the albumin such as sodium Pentothal, decreases. So the dose of the drug should be decreased. ${ }^{6,9}$

On the other hand, some drugs will need an increase in dose due to an increase in the volume of distribution, which may be the result of edema and increased level of gammaglobulines. ${ }^{4}$

With respect to the increased risk of post-operative delirium and hepatic encephalopathy, use of long acting narcotics and sedatives in patients with liver failure is not recommended. Patients with hepatic failure are at a risk of hepatotoxic reaction with benzodiazepines and barbiturates. In these cases, a cholestatic pattern of liver damage is expected. Patients may present with fever, hepatitis, eosinophilia, and dermatitis. ${ }^{6}$ 
One of the most important drug selections for patients with liver failure, is selection of proper anesthetic drug for maintenance of anesthesia. As we know today, volatiles such as sevoflurane or isoflurane not only can be harmless but also useful and beneficial for disease prognosis because of debatable hepatoprotective role especially in ischemic scenarios. ${ }^{10}$

Remembering that halothane can cause lethal hepatitis in patients who undergo general anesthesia, this drug should be avoided and novel volatiles such as desflurane is recommended. ${ }^{11}$

Because of altered pharmacodynamic properties of drugs, it is logical to use advanced monitoring devices to monitor drug effects and avoid the excessive use of anesthetic medications. In this manner titration of drugs against effects would be very beneficial. ${ }^{6,8}$

\section{CONCLUSION}

Patients with underlying liver disease have an increased surgical risk. Prediction of surgical risk is based on the degree of liver dysfunction, the type of surgery, and the preoperative clinical status of the patient as previously mentioned. Management of surgical diseases in patients with cirrhosis, similar to any other complicated organ failure, needs a close cooperation between gastroenterologists, anesthesiologists, and surgeons to predict and manage any potential risk before leading to serious complications.

\section{ETHICAL APPROVAL}

There is nothing to be declared.

\section{CONFLICT OF INTEREST}

The author declares no conflict of interest related to this work.

\section{REFERENCES}

1. Efeoglu C, Sipahi Calis A, Karasu Z, Koca H, Boyacioglu $\mathrm{H}$. Prospective randomized single-blind study of post-operative bleeding after minor oral surgery in patients with cirrhosis. Turk J Gastroenterol. 2019;30:171-176. doi: 10.5152/tjg.2018.18078.

2. Malik SM, Ahmad J. Preoperative risk assessment for patients with liver disease. Med Clin North Am 2009;93:917-29, ix. doi: 10.1016/j.mcna.2009.03.001.

3. Sabate A, Acosta Villegas F, Dalmau A, Koo M, Sansano Sanchez T, Garcia Palenciano C. [Anesthesia in the patient with impaired liver function]. Rev Esp Anestesiol Reanim 2011;58:574-81.

4. Rahimzadeh P, Safari S, Faiz SH, Alavian SM. Anesthesia for patients with liver disease. Hepat Mon 2014;14:e19881. doi: 10.5812/hepatmon.19881.

5. Wiklund RA. Preoperative preparation of patients with advanced liver disease. Crit Care Med 2004;32(4 Suppl):S106-15.

6. Friedman L. Effects of anesthesia and surgery on the liver. Up To Date Waltham (MA): UpToDate; 1992. 2015;Last updated 2015.(Available from: www.UpToDate.com).

7. Lentschener C, Ozier Y. Anaesthesia for elective liver resection: some points should be revisited. Eur J Anaesthesiol 2002;19:780-8.

8. Moreau R, Hadengue A, Soupison T, Mamzer MF, Kirstetter P, Saraux JL, et al. Arterial and mixed venous acidbase status in patients with cirrhosis. Influence of liver failure. Liver 1993;13:20-4.

9. Soleimanpour H, Safari S, Shahsavari Nia K, Sanaie S, Alavian SM. Opioid Drugs in Patients With Liver Disease: A Systematic Review. Hepat Mon 2016;16:e32636. doi: 10.5812/hepatmon.32636.

10. Mohseni M, Safari S, Alavian SM. Volatile anesthetics in ischemic liver injury: enemy or friend? Hepat Mon 2014;14:e19880. doi: 10.5812/hepatmon.19880.

11. Habibollahi P, Mahboobi N, Esmaeili S, Safari S, Dabbagh A, Alavian SM. Halothane-induced hepatitis: A forgotten issue in developing countries: Halothane-induced hepatitis. Hepat Mon 2011;11:3-6. 